\title{
A Murine Model of a Burn Wound Reconstructed with an Allogeneic Skin Graft
}

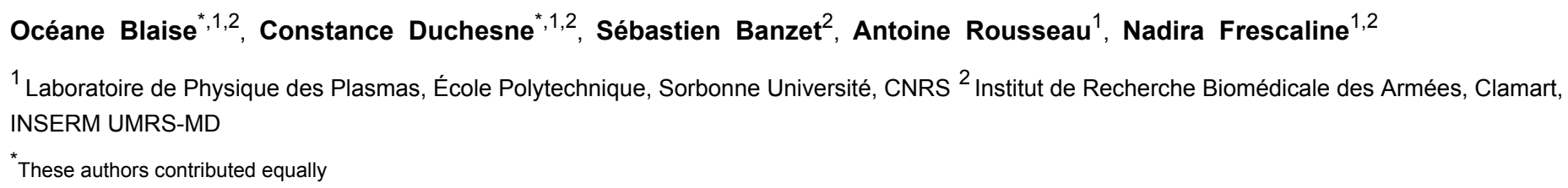

\section{Corresponding Author}

Nadira Frescaline

nadira.frescaline@Ipp.polytechnique.fr

\section{Citation}

Blaise, O., Duchesne, C., Banzet, S., Rousseau, A., Frescaline, N. A Murine Model of a Burn Wound Reconstructed with an Allogeneic Skin Graft. J. Vis. Exp. (162), e61339, doi:10.3791/61339 (2020).

\section{Date Published}

August 8, 2020

DOI

$10.3791 / 61339$

URL

jove.com/video/61339

\section{Abstract}

Trivial superficial wounds heal without complications by primary intention. Deep wounds, such as full thickness burns, heal by secondary intention and require surgical debridement and skin grafting. Successful integration of the donor graft into a recipient wound bed depends on timely recruitment of immune cells, robust angiogenic response and new extracellular matrix formation. The development of novel therapeutic agents, which target some key processes involved in wound healing, are hindered by the lack of reliable preclinical models with optimized objective assessment of wound closure. Here, we describe an inexpensive and reproducible model of experimental full thickness burn wound reconstructed with an allogeneic skin graft. The wound is induced on the dorsum surface of anaesthetized inbred wild type mice from the BALB/C and SKH1-Hrhr backgrounds. The burn is produced using a brass template measuring $10 \mathrm{~mm}$ in diameter, which is preheated to 80 ${ }^{\circ} \mathrm{C}$ and delivered at a constant pressure for $20 \mathrm{~s}$. Burn eschar is excised 24 hours after the injury and replaced with a full thickness graft harvested from the tail of a genetically similar donor mouse. No specialized equipment is required for the procedure and surgical techniques are straightforward to follow. The method may be effortlessly implemented and reproduced in most research settings. Certain limitations are associated with the model. Due to technical difficulties, the harvest of thinner split thickness skin grafts is not possible. The surgical method we describe here allows for the reconstruction of burn wounds using full thickness skin grafts. It may be used to carry out preclinical therapeutic testing.

\section{Introduction}

Surgical debridement and skin grafting are common clinical practices used in the management of chronic wounds ${ }^{1}$, burn wounds $^{2}$, and acute wounds such as traumatic wounds ${ }^{3}$. Skin grafting refers to the surgical procedure, which involves 
the removal of healthy skin from one part of the body and transferring it to another. Donor grafts replace the lost tissue and provide a structural scaffold for cellular migration and growth. Following integration into the recipient site, skin grafts replace the lost skin barrier by providing protection from microbial invasion, harmful effects of the external environment and excessive loss of moisture ${ }^{4}$. Successful skin graft integration depends on several factors. These include adequate immune responses in the presence of microbial infections and timely resolution of inflammation, robust angiogenesis at the wound site and establishment of vascular anastomoses between the recipient bed and the donor graft ${ }^{5}$. As the graft begins to degrade, resident dermal cells must be replaced by cells capable of producing new extracellular matrix. At the same time, the epidermal keratinocytes must crawl over the newly produced matrix to form the neo-epidermis and re-epithelialize the wound. It is, therefore, evident that efficient migration of cells from the recipient bed into the donor graft is another determining factor that influences successful graft incorporation. Given the vast number of factors involved in wound healing ${ }^{6}$, which may be impossible to control in the human trials due to ethical limitations, models of pre-clinical experimental skin grafting are necessary. Development of pre-clinical models of burn wound healing and associated skin grafting will be important for understanding of complex mechanisms involved in cutaneous tissue repair and essential for the testing of new therapeutic agents. The in vitro models of wound healing are unable to accurately mimic the complexity of the cutaneous tissue. The in vivo animal models are an indispensable investigative tool in understanding the mechanisms involved in tissue repair.

Several methods of skin grafting technique were developed in rodents to mimic surgical excision and burn wound reconstruction ${ }^{7,8,9}$. However, most of the previously described procedures failed to induce a thermal burn injury prior to skin grafting. Instead of the burn wound, a full thickness excisional wound was induced, which was then reconstructed with a full thickness skin allograft ${ }^{7}$. Various anatomical landmarks such as the ear, tail and back have been used for harvesting of the donor skin in rodents ${ }^{7}, 8$. Different graft fixation and stabilization techniques were reported, including a "no suture technique" ${ }^{\prime}$, sutures ${ }^{7}$ and surgical glue $10,11,12$.

The purpose of this study was to develop a murine model of a full thickness burn wound that would recapitulate the current gold standard approach in burn treatment, which involves nonviable tissue excision and skin grafting. A thermal burn was induced on the dorsum surface of a mouse using a preheated brass template. Burn eschar was excised and replaced with a full thickness graft harvested from the tail of a donor mouse. There are three key advantages to this experimental model. First, more than one burn wound may be induced on the back of the recipient mouse, and four donor skin grafts may be harvested from a single tail of the donor mouse. This means that several experimental and control treatments may potentially be compared using the same recipient and donor animals. Depending on the desired route of administration, the control treatment may include local or systemic administration of the vehicle or placebo control (e.g., topical application of ointment, subcutaneous, intraperitoneal or intravenous injection of solution). Second, timing of the treatment and the endpoint of the experiment may be controlled. Third, this model depends on the reconstruction of wounds using full thickness grafts harvested from the tail, which are known to have a higher probability for successful incorporation into the donor site compared to the skin harvested from the back ${ }^{13}$. This may be due to the lower 
number of epidermal Langerhans cells, which play a key role in cutaneous immunobiology, and are associated with the skin graft rejection $^{14}$.

The proposed model of wound healing and graft integration may well be applied to transgenic and knockout mice. The use of genetically modified mice will assist in elucidating the roles that certain genes may play during wound repair. Exogenous application of topical wound preparations or subcutaneous administration of therapeutic antibodies at the site of the injury may also be considered.

Due to technical difficulties, split thickness skin grafts consisting of the epidermis and part of the dermis are difficult to obtain in mice. Full thickness skin grafts consisting of the epidermis and full thickness dermis are known to require a well-vascularized wound bed for successful integration. The inability to harvest split thickness skin grafts in mice may be regarded as a limitation of this model. The fixation of the skin graft to the recipient wound bed was achieved via the application of the surgical adhesive glue, which is associated with less trauma and rapid degradation compared to other means of tissue fixation ${ }^{15}$. Previous studies have shown that suturing is associated with stronger tissue fixation than the surgical glue at $24 \mathrm{~h}$ after the surgical procedure ${ }^{15}$, which may be considered as a disadvantage of the procedure. However, at later timepoints, the biomechanical strength of wounds treated with a surgical adhesive becomes comparable to sutures $^{15}$ and better than staple fixation ${ }^{16}$. Following tissue fixation with the surgical glue, wounds must be covered with a wound dressing. Although wounds on the dorsal surface of the mouse are difficult for the animal to reach, the wound dressing, on the other hand, is easy for the animal to manipulate and remove. Frequent wound dressing changes may be warranted.
Anesthesia-induced hypothermia in small rodents is a welldocumented phenomenon ${ }^{17}$. Hypothermia is a side effect of this procedure, which causes complications, and potentially compromises both animal health and data quality. Therefore, this method warrants the implementation of temperature management strategies, especially if hairless SKH1-Hrhr are used.

The most significant limitation of using mice to mimic human wound closure is the difference between the skin anatomy and physiology. Mouse wounds heal mostly via contraction, whereas human wounds heal through granulation tissue formation and re-epithelialization ${ }^{18}$. To account for this discrepancy, the current model may be modified and used in combination with a splinting ring tightly adhered around the wound to prevent skin contraction ${ }^{19}$. Given some advantages and disadvantages of this in vivo protocol, this model could serve as a tool to study certain processes involved in wound healing that are impossible to study in vitro.

\section{Protocol}

All experiments were approved by the French Department of Higher Education and Research (Study Number: 122162017111616517670v2 and DAP180012). All mice were single-housed upon arrival in plastic cages and were allowed a 7-day acclimatization period prior to the study. The animal room was maintained at a $12 / 12 \mathrm{~h}$ light/dark cycle (lights on at 07:00). Food and tap water were provided ad libitum. BALB/c and SKH1-Hrhr mice were fed traditional wheat/soybased diet. Sawdust bedding was provided along with nesting material. 


\section{Equipment preparation}

1. Prepare a burning device for the procedure and set it to $80^{\circ} \mathrm{C}$ using the temperature controller (Figure 1A). Verify the temperature of the brass template (Figure 1B,C) using the infrared thermal imaging camera.

2. Ensure that the digital manometer is operating correctly.

3. Cover the stage with a surgical drape and adjust the height of the table (Figure 1A).

\section{Pre-operative and intra-operative animal care}

1. Acquire BALB/c and SKH1-Hrhr mice, 6-8 weeks of age.

2. Add paracetamol suspension at $3 \mathrm{mg} / \mathrm{mL}$ to drinking water and supply 12 hours before and up to 72 hours after the procedure.

3. Using a $1 \mathrm{~mL}$ syringe and a $26 \mathrm{G}$ needle, administer buprenorphine subcutaneously at $0.05 \mu \mathrm{g} / \mathrm{g} 30 \mathrm{~min}$ before the procedure and every 6 hours for the first 72 hours after the procedure.

4. Using a $1 \mathrm{~mL}$ syringe and a $26 \mathrm{G}$ needle, administer lidocaine to the dorsum of the mouse and 2-3 mm distal to the area of the burn wound. Inject lidocaine at $0.05 \mu \mathrm{g} /$ g subcutaneously 15 min before the induction of the burn wound.

5. Anesthetize mice using an intraperitoneal injection of xylazine at $10 \mathrm{mg} / \mathrm{kg}$ and ketamine $100 \mathrm{mg} / \mathrm{kg}$. Use a 1 $\mathrm{mL}$ syringe and a $26 \mathrm{G}$ needle to administer the injection.

6. Critical step: Place the anaesthetized mouse on a heated pad and keep the mouse warm to prevent hypothermia for the first 30 minutes after the induction of anesthesia and for at least 15 minutes after the recovery from anesthesia. In addition to the heated pad, other modalities including heat lamps, circulating warm liquids or air, and prewarmed heat reservoirs may be used to regulate the body temperature.

7. Apply lubrication gel on the eyes of the mouse to prevent dehydration of the cornea.

8. Use the toe pinch withdrawal reflex to assess the depth of anesthesia.

9. Using a $1 \mathrm{~mL}$ syringe and a $26 \mathrm{G}$ needle administer 200 $\mu \mathrm{L}$ of Lactated Ringer's Solution supplemented with $5 \%$ dextrose. Administer fluid replacement subcutaneously immediately after the induction of anesthesia and 6 hours after the procedure to prevent dehydration.

\section{Full thickness burn wound induction}

1. Shave the anaesthetized mouse with the hair clippers.

2. Apply depilating cream on the dorsum surface of the mouse for 1 minute. Wipe off the cream using some sterile gauze and clean the area with a piece of damp gauze. Blot the skin with some gauze until dry.

3. Place the mouse on the stage covered with a surgical drape and move the stage upward closer to the preheated brass template.

4. Apply the circular brass template on the back of the mouse $\left(80{ }^{\circ} \mathrm{C}\right.$ for $\left.20 \mathrm{~s}\right)$ using constant pressure of 0.15 $\mathrm{N}$ (Figure 2).

5. Critical step: Immediately after the burn induction, place the anaesthetized animal on the heated pad to prevent hypothermia and keep the mouse warm during and after the procedure. Once recovered from anesthesia, return the mouse back to the cage.

6. Critical step: Provide a mashed diet on the cage floor for the first 72 hours after the surgical procedure. Mice are 
sometimes reluctant to reach up to a sipper tube to drink water after a burn wound injury.

\section{Harvesting of the donor graft}

1. Make a longitudinal incision with a scalpel in the upper part of the tail of the donor mouse and gently remove the skin using surgical forceps.

2. Place the tail skin into a sterile Petri dish filled with 10 $\mathrm{mL}$ of sterile $0.9 \%$ saline solution. Use a ruler to measure out individual grafts and cut the tail skin into pieces, each measuring $15 \mathrm{~mm}$, using a scalpel.

3. Once prepared, keep the skin grafts in $0.9 \%$ saline solution at $4{ }^{\circ} \mathrm{C}$ for up to 2 hours.

\section{Surgical excision and skin grafting}

1. Twenty-four hours after the burn induction, prepare the mouse for anesthesia by inhalation of isoflurane. Place the mouse into the induction chamber and induce anesthesia using $5 \%$ isoflurane in $100 \%$ oxygen at a flow rate of $4 \mathrm{~L} / \mathrm{min}$. To maintain anesthesia during surgery, use $2 \%$ isoflurane at $2 \mathrm{~L} / \mathrm{min}$.

2. Place a surgical drape on the mouse and cut out a window to expose the surgical field. Using the aseptic technique, swab the wound first with povidone-iodine and then with $70 \%$ alcohol.

3. Gently pick up the burned tissue with a pair of surgical tweezers and excise all necrotic and nonviable tissue with sterile surgical scissors and tweezers. Remove the panniculus carnosus layer of the hypodermis to create a stable recipient bed.

4. Place the skin graft on the freshly prepared wound bed. Gently pull the surrounding skin toward the skin graft using surgical tweezers. Apply some surgical adhesive to attach the graft to the wound bed and gently press to align the skin edges. Critical step: The size of the wound bed must be slight larger than the size of the skin graft to ensure successful engraftment.

5. Allow the mouse to recover from anesthesia. Critical step: Place the mouse on a heated pad. Keep the mouse warm during and after the procedure to prevent hypothermia.

6. Apply inert paraffin gauze dressing and adhesive secondary dressing over the grafted wound.

7. Place the mouse into an individual cage. Critical step: Provide some mashed diet on the cage floor for the first 72 hours after the surgical procedure and monitor daily.

8. Provide toys and enrich the environment.

\section{Digital imaging and post-mortem wound collection}

1. Photograph wounds with a digital camera by placing a ruler next to the wound (Figure 3).

2. At the end point of the experiment, euthanize animals by exposure to carbon dioxide and cervical dislocation. Critical step: Excessive pulling action of the skin during the cervical dislocation may damage the graft.

3. At post-mortem, surgically excise the dorsal burn wounds to the fascia using scissors. Bisect wounds. Fix half in $10 \%$ buffered formalin and process for histology and immunohistochemistry. Fast freeze the other half in liquid nitrogen for RNA extraction and protein quantitation and keep at $-80^{\circ} \mathrm{C}$. 


\section{Skin histology, immunohistochemistry and collagen visualization}

1. Embed samples of skin into paraffin, cut to $4 \mu \mathrm{m}$ sections and place onto positively charged slides.

2. Use slides stained with hematoxylin and eosin to evaluate the rate of re-epithelialization (\% of the original wound). The area of the wound that is covered with neo-epidermis may be expressed as a percentage of the entire wound (Figure 4). Use a digital microscope application and ImageJ software to perform the microscopic analysis of the sections.

3. Use histological sections ( $4 \mu \mathrm{m}$ thickness) prepared from formalin-fixed and paraffin-embedded tissue and subject them to immunohistochemistry.

4. To assess collagen $I$ and fibronectin expression in wounds, apply primary antibodies and incubate for 1 h. Detection may be performed by species-specific horseradish peroxidase (HRP) or alkaline phosphatase (AP)-conjugated secondary antibodies.

5. React sections with either: (i) HRP,3,3'-diaminobenzidine (DAB) or (ii) AP, Bond Polymer Refine Red (Table of Materials), which yields a bright red color (Figure 5). Scan the sections using an instrument and analyze with the digital microscope application and ImageJ.

6. To enable histological assessment of collagen deposition, perform trichrome staining on histological sections using a commercial kit.

7. For collagen fiber visualization, use multiphoton microscopy and second harmonic generation technique (Figure 5). Use a multiphoton microscope for tissue imaging as previously described ${ }^{20}$. Use a Ti:Sapphire laser with a center wavelength at $810 \mathrm{~nm}$ as the laser source for generating second harmonic and two-photon excited fluorescence signals (TPEF).

8. Use a laser beam equipped with a $25 \mathrm{x} / 0.95 \mathrm{~W}$ objective to collect and excite second harmonic generation (SHG) and TPEF. Detect signals as described previously ${ }^{21}$ by NDD PMT detectors. Use software for laser scanning control and image acquisition.

\section{Representative Results}

The results demonstrate that the protocol developed is a straightforward method, which permits the induction of a full thickness burn wound in mice. Burns are induced using a preheated brass template (Figure 1A-C). The burned area appears as a circular wound with a white eschar and a hyperemic zone. The size of the burn wound is slightly larger at 24 hours after the burn injury as a result of the welldescribed phenomenon known as the burn injury progression, which is possibly due to acute inflammation ${ }^{22}$. After excision, burn wounds are reconstructed using allogeneic skin graft (Figure 3). On day 7 after the burn injury, wounds become vascularized ${ }^{5}$, which is an indication of successful engraftment. Epidermal keratinocytes migrate from the adjacent recipient skin in the effort to close the wound and bridge the gap between the two edges of the wounds. Microscopic analysis of $\mathrm{H}$ and $\mathrm{E}$ stained section of wounds revealed that the length of the neo-epidermis becomes significantly longer on day 7 after burn injury compared to day 3 after burn injury (Figure 4B). Prior to performing a large study, it is highly recommended that researchers complete a pilot study, which enables the exploration of a novel intervention, assessment of feasibility, identification of modifications to the method to ensure reproducibility. Statistically significant effects are difficult to detect in smaller samples, whereas increasing the sample size is one way to 
boost the statistical power of a test. For example, to detect a statistically significant difference $(p<0.05)$ in the rate of wound re-epithelialization (Figure 4) between groups, the sample size should be between six and eight mice per group. All experiments should be repeated at least twice. As matrix producing cells, such as fibroblasts, migrate from the recipient tissue into the graft, key components of the extracellular matrix, including collagen I and fibronectin become highly expressed in the newly formed matrix (Figure 5).

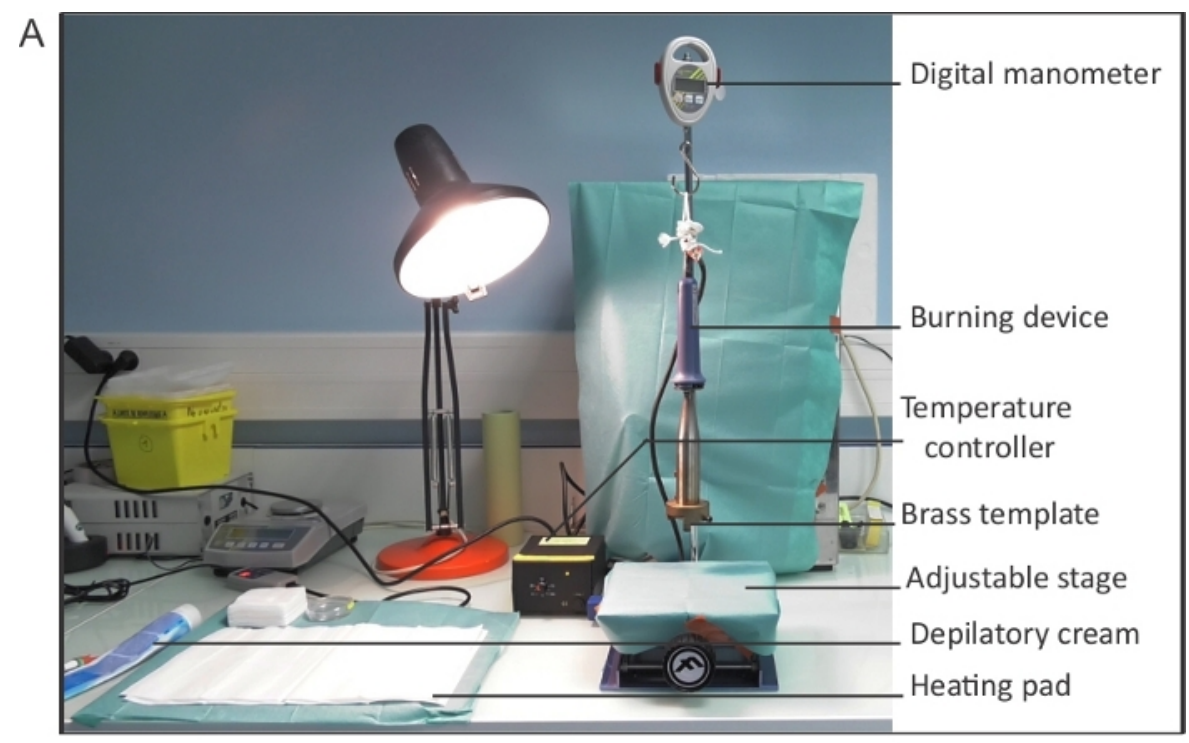

B

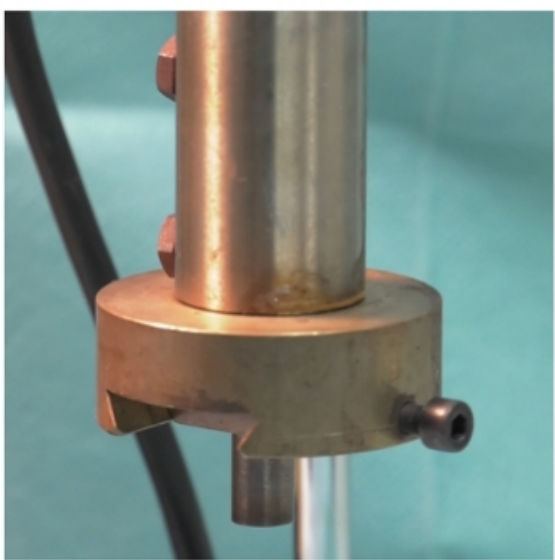

C

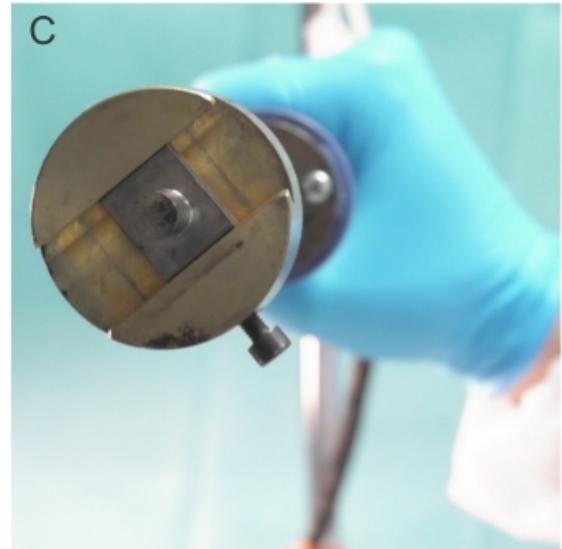

Figure 1: Burning device setup. (A) Placement and set-up of the burning device. The burning device is connected to the temperature controller and is attached to the digital monometer to enable the monitoring of pressure. The burning device is suspended above an adjustable stage - flat surface onto which mice are placed for the induction of burn. (B-C) A close up image of the brass template used to induce wound burns. $(\mathbf{C})$ The diameter of the brass template is $1 \mathrm{~cm}$. Please click here to view a larger version of this figure. 


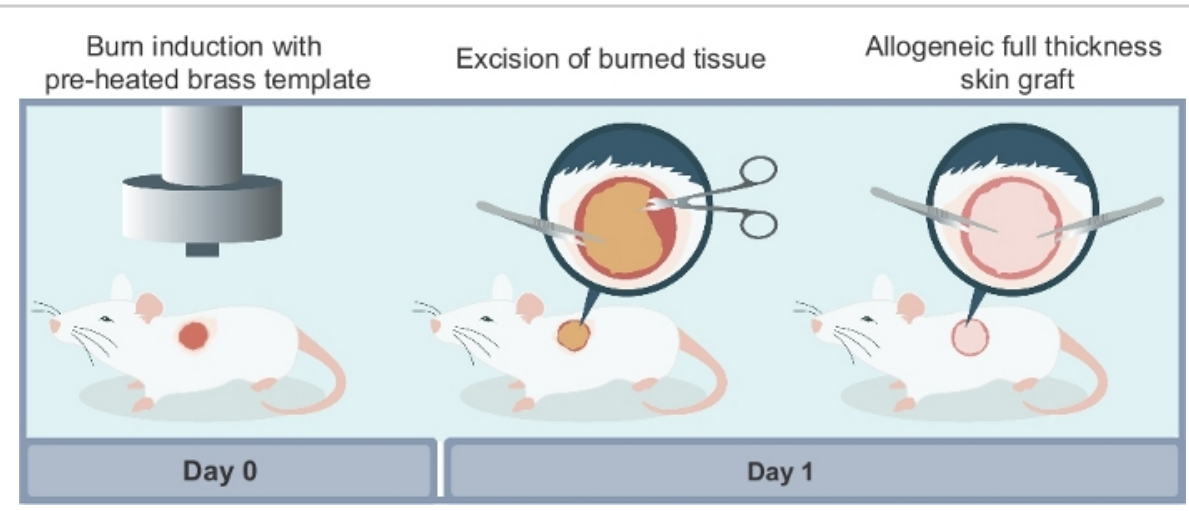

Figure 2: Schematic illustration of the different steps required to reproduce the experimental model described in this article. There are three main steps to the procedure: (i) induction of the burn wound using a preheated brass template; (ii) surgical excision of the non-viable necrotic tissue at 24 hours after the burn injury; (iii) surgical wound reconstruction using a full thickness allogeneic skin graft harvested from a donor mouse. Please click here to view a larger version of this figure.

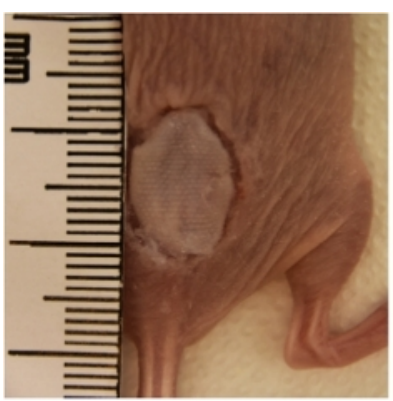

Day 1

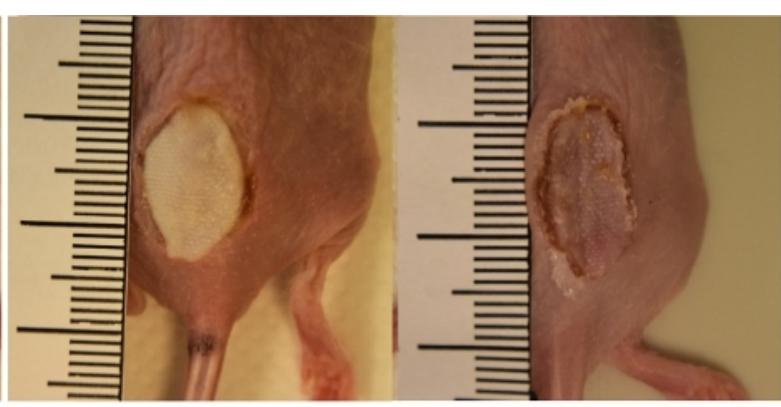

Day 7

Figure 3: The macroscopic view of reconstructed mouse burn wounds. Representative digital images of burn wounds reconstructed with allogeneic skin grafts on days $0,1,3$ and 7 after burn injury. The ruler on images is in millimeters. Please click here to view a larger version of this figure. 

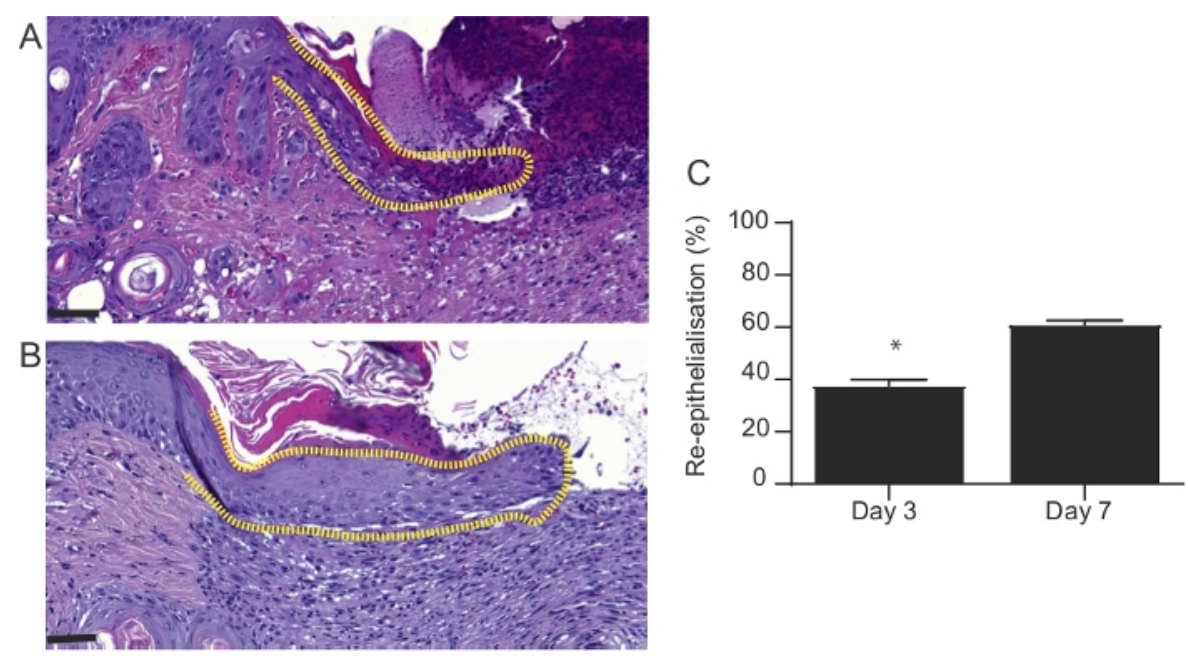

Figure 4: Microscopic appearance of wounds at 3 and 7 days after burn injury. H\&E-stained sections of wounds 3 and 7 days after burn injury. The length of neo-epidermis (dotted line) is significantly increased in (A) day 3 wounds compared to (B) day 7 wounds. In (A) and (B), the scale bar is $100 \mu \mathrm{m}$. (C) Graphical representation of the percentage of wound reepithelialization. This was evaluated by measuring the length of neo-epidermis at day 3 and 7 post-burn injury and expressed as a percentage of the whole wound length. Results represent mean \pm S.E.M. ( $n=6$ mice in day 3 group; $n=6$ mice in day 7 group, ${ }^{*} p<0.05$; Student's t-test). Please click here to view a larger version of this figure. 

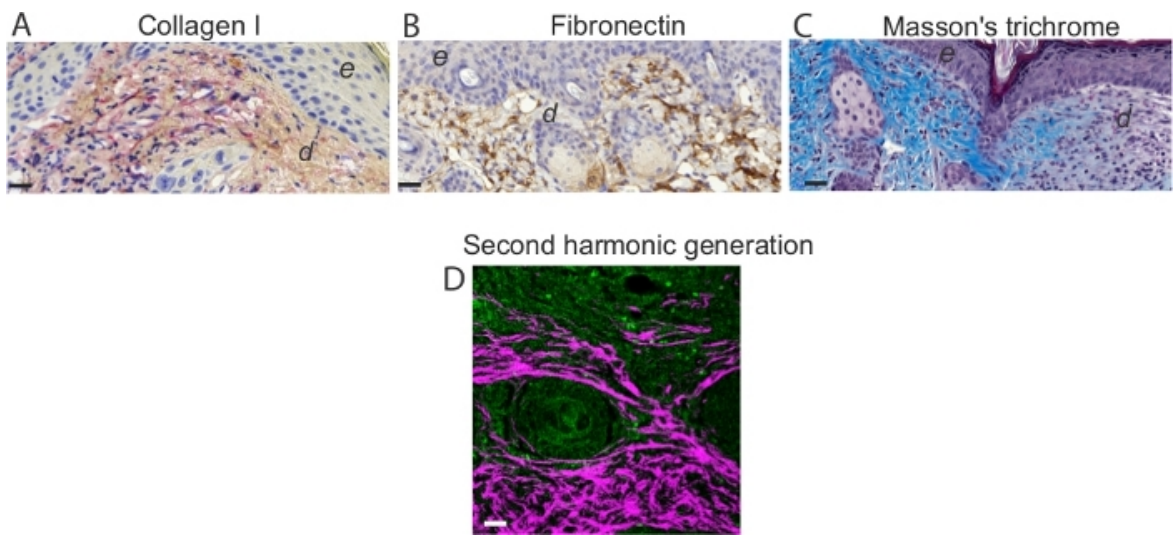

Figure 5: Assessment of the extracellular matrix and collagen I visualization. Representative images of immunohistochemistry analysis on day 7 mouse wounds stained for (A) collagen and (B) fibronectin. Note intense red staining in elongated spindle-shaped collagen I-positive cells. Note brown staining in fibronectin-positive cells in the dermis of day 7 wounds. Scale bar $=50 \mu \mathrm{m}$ in all images. In (A) and (B), e denotes epidermis and d denotes dermis. (C) Visualization of collagen fibers and histological assessment of collagen deposition. (D) Representative TPEF/SHG collagen image of day 7 mouse wounds. Simultaneous TPEF/SHG acquisition using circular polarization and SHG signals were selectively processed to obtain a binary distribution of SHG after applying a threshold. TPEF images (green) and SHG images (white) were pseudo-colored and overlaid. Scale bar $=50 \mu \mathrm{m}$. Please click here to view a larger version of this figure.

\section{Discussion}

According to the thickness classification of burn injury ${ }^{23}$, full thickness burns are characterized by evident involvement of the whole thickness of the skin and certain portion of the subcutaneous tissue. This type of wound can heal only by contraction or with skin grafting ${ }^{2}$. An inherent limitation of the method described in this article is that only full thickness grafts, as opposed to the split thickness grafts, which are often used in the clinical setting, were harvested from the tail of a mouse. This was due to the technical difficulty, as the mouse skin is too thin to obtain split thickness grafts. It must be pointed out that full thickness grafts require a well vascularized wound bed, whereas split thickness skin grafts are able to survive at donor sites with less vascularity ${ }^{24}$. Previous studies showed that a burn wound induced on the back of the mouse was associated with a robust formation of new vasculature ${ }^{5}$. This suggests that a well vascularized area, such as the dorsum of the mouse, could be considered as the anatomical landmark for the induction of burn wounds.

Burn wound depth is an important factor to consider. The depth of the burn wound must be consistent between individual mice. The reproducibility of the burn wound depth depends on the temperature of the brass template, pressure and duration of the heat exposure. The burn wound depth must be verified histologically. It is important to keep in mind that excessive pressure or prolonged exposure of the skin to the preheated brass template may injure the underlying tissue. The tissue surrounding the vertebral column, including the components of the central and peripheral nervous system, 
are sensitive to heat, and if damaged may result in hind leg paralysis.

Although no postoperative mortality was directly associated with the surgical procedure, a small number of hairless SKH1-Hrhr mice, which are especially sensitive to cold, developed hypothermia and failed to recover after the general anaesthesia. Therefore, supplementary heat must be provided during all aesthetic events and constant surveillance is required while the mouse is anaesthetized.

The method described in this study was not associated with the surgical site infection. However, aseptic technique must be used to prevent the transfer of microorganisms into the surgical wound during the perioperative period. Inoculation of the wound with bioluminescent or fluorescent microorganisms may be incorporated into the procedure. This technique may be useful in studying infectious organisms and their pathogenesis ${ }^{25}$. For example, exogenous addition or injection of bioluminescent bacteria, may permit the monitoring of the microbial burden using the in vivo whole animal imaging ${ }^{25}$. Given that mouse hair is known to interfere with the in vivo whole animal fluorescence and bioluminescence imaging, hairless SKH1-Hrhr mice are ideal hosts for the studies involving fluorescent or bioluminescent reporters.

Wound tissue samples may be collected at different time points and processed for histological and immunohistochemical analysis. Protein and RNA may be isolated from the skin biopsy and molecular biology techniques may be used to assess the expression of key molecules involved in wound healing.

In the present study, we described an experimental model of burn wound healing and allogeneic skin engraftment.
This procedure can be modified and serve as a model for preclinical studies.

\section{Disclosures}

The authors declare that they have no competing financial interests.

\section{Acknowledgments}

This work was supported by La Direction Générale de L'Armement, l'Agence de l'Innovation de Défense and École Polytechnique. We thank our colleague Mr Yann Plantier from École Polytechnique who provided insight and expertise that greatly assisted the production of the video file. The authors thank Mr Benoit Peuteman and Ms Charlotte Auriau from INSERM Lavoisier (SEIVIL) US 33, Hôpital Paul Brousse, Villejuif for their animal well-being and care expertise provided during the course of this project.

\section{References}

1. Shakir, S. et al. Indications and Limitations of Bilayer Wound Matrix-Based Lower Extremity Reconstruction: A Multidisciplinary Case-Control Study of 191 Wounds. Plastic and Reconstructive Surgery. (2019).

2. Greenhalgh, D. G. Management of Burns. New England Journal of Medicine. 380 (24), 2349-2359 (2019).

3. Bosse, M. J. et al. An analysis of outcomes of reconstruction or amputation after leg-threatening injuries. New England Journal of Medicine. 347 (24), 1924-1931 (2002).

4. Braza, M. E., Fahrenkopf, M. P. in StatPearls. (2019).

5. Duchesne, C., Banzet, S., Lataillade, J. J., Rousseau, A., Frescaline, N. Cold atmospheric plasma modulates endothelial nitric oxide synthase signalling and enhances 
burn wound neovascularisation. Journal of Pathology. 249 (3), 368-380 (2019).

6. Eming, S. A., Martin, P., Tomic-Canic, M. Wound repair and regeneration: mechanisms, signaling, and translation. Science Translational Medicine. 6 (265), 265sr266 (2014).

7. Pakyari, M. et al. Local Expression of Indoleamine 2,3, Dioxygenase Prolongs Allogenic Skin Graft Take in a Mouse Model. Advances in Wound Care (New Rochelle). 8 (2), 58-70 (2019).

8. Pakyari, M. et al. A new method for skin grafting in murine model. Wound Repair and Regeneration. 24 (4), 695-704 (2016).

9. McFarland, H. I., Rosenberg, A. S. Skin allograft rejection. Current Protocols in Immunology. Chapter 4, Unit 44 (2009).

10. Cristobal, L. et al. Local Growth Hormone Therapy for Pressure Ulcer Healing on a Human Skin Mouse Model. International Journal of Molecular Sciences. 20 (17) (2019).

11. Melican, K., Aubey, F., Dumenil, G. Humanized mouse model to study bacterial infections targeting the microvasculature. Journal of Visualized Experiments. (86) (2014).

12. Racki, W. J. et al. NOD-scid IL2rgamma(null) mouse model of human skin transplantation and allograft rejection. Transplantation. 89 (5), 527-536 (2010).

13. Larsen, C. P. et al. Migration and maturation of Langerhans cells in skin transplants and explants. Journal of Experimental Medicine. 172 (5), 1483-1493 (1990).

14. Leonard, D. A., Kurtz, J. M., Cetrulo, C. L., Jr. Vascularized composite allotransplantation: towards tolerance and the importance of skinspecific immunobiology. Current Opinion in Organ Transplantationt. 18 (6), 645-651 (2013).

15. Stoikes, N. et al. Biomechanical evaluation of fixation properties of fibrin glue for ventral incisional hernia repair. Hernia: The Journal of Hernias and Abdominal Wall Surgery. 19 (1), 161-166 (2015).

16. Foster, K. et al. Efficacy and safety of a fibrin sealant for adherence of autologous skin grafts to burn wounds: results of a phase 3 clinical study. Journal of Burn Care \& Research. 29 (2), 293-303 (2008).

17. Caro, A. C., Hankenson, F. C., Marx, J. O. Comparison of thermoregulatory devices used during anesthesia of C57BL/6 mice and correlations between body temperature and physiologic parameters. Journal of the American Association for Laboratory Animal Science. 52 (5), 577-583 (2013).

18. Grada, A., Mervis, J., Falanga, V. Research Techniques Made Simple: Animal Models of Wound Healing. Journal of Investigative Dermatology. 138 (10), 2095-2105 e2091 (2018).

19. Wang, X., Ge, J., Tredget, E. E., Wu, Y. The mouse excisional wound splinting model, including applications for stem cell transplantation. Nature Protocols. 8 (2), 302-309 (2013).

20. Ruzehaji, N. et al. Pan PPAR agonist IVA337 is effective in prevention and treatment of experimental skin fibrosis. Annals of the Rheumatic Diseases. 75 (12), 2175-2183 (2016)

21. Ruzehaji, N. et al. Combined effect of genetic background and gender in a mouse model of bleomycin-induced skin fibrosis. Arthritis Research \& Therapy. 17145 (2015). 
22. Singer, A. J., Boyce, S. T. Burn Wound Healing and Tissue Engineering. Journal of Burn Care \& Research. 38 (3), e605-e613 (2017).

23. Shakespeare, P. Burn wound healing and skin substitutes. Burns. 27 (5), 517-522 (2001).

24. Sun, B. K., Siprashvili, Z., Khavari, P. A. Advances in skin grafting and treatment of cutaneous wounds. Science. 346 (6212), 941-945 (2014).

25. Miller, R. J. et al. Development of a Staphylococcus aureus reporter strain with click beetle red luciferase for enhanced in vivo imaging of experimental bacteremia and mixed infections. Scientific Reports. 9 (1), 16663 (2019). 